\title{
Avaliação de Desempenho de Placas de Rede 10GbE: Uma Estratégia Baseada em Análise de Sensibilidade
}

\author{
Paulo Henrique Rocha e Francisco Airton Silva \\ ${ }^{1}$ Universidade Federal do Piauí (UFPI) \\ Picos - PI - Brazil \\ paulohenriquegrocha@gmail.com, faps@ufpi.edu.br
}

\begin{abstract}
Computers have network cards that are mandatory electronic components to perform communication between machines. The 10GbE board is one of the boards most commonly used by Internet companies. Package capture frameworks are used to process large quantities of packages without discarding them. Network infrastructures managers do not have at their disposal studies that help them in deciding which board and framework to adopt according to their needs. The present work presents a sensitivity analysis evaluating the impact of two network card brands with $10 \mathrm{GbE}$ capacity and two packet capture frameworks. Different combinations were indicated making this work an aid tool for network professionals.
\end{abstract}

Resumo. Os computadores possuem placas de rede que são componentes eletrônicos obrigatórios para realizar a comunicação entre máquinas. A placa de 10GbE é uma das placas mais utilizadas por companhias provedores de Internet. Os frameworks de captura de pacotes são utilizados a fim de processarem grandes quantidades de pacotes, sem descartá-los. Gerentes de infraestruturas de redes não possuem ao seu alcançe estudos, que os auxiliem na tomada de decisão de qual placa e framework adotar de acordo com as suas necessidades. O presente trabalho apresenta uma análise de sensibilidade avaliando o impacto de duas marcas de placas de rede com capacidade $10 \mathrm{GbE}$ e dois frameworks de captura de pacotes. Diferentes combinações foram indicadas tornando este trabalho uma ferramenta de auxilio para os profissionais de rede.

\section{Introdução}

O mercado domiciliar de internet no Brasil é atendido prioritariamente pelas pequenas empresas provedoras de acesso à Internet: 94\% daquelas com 1 a 9 pessoas ocupadas, e $92 \%$ e $91 \%$ daquelas com 10 a 19 e 20 a 49, respectivamente (entre os provedores de grande porte, aqueles com 250 a 499 e mais de 500 pessoas ocupadas, $56 \%$ e $52 \%$, respectivamente, atendem esse mercado) [Cetic.br 2016].

Os pequenos e médios provedores cada vez mais precisam ir em busca de equipamentos melhores, na qual apresentam altos custos de aquisição. Portanto encontrar soluções mais baratas e que atendam suas demandas de forma mais eficiente é um dos problemas enfrentados por essas empresas. As interfaces de rede (NIC) com taxas de dados de $1 \mathrm{GbE}$ e $10 \mathrm{GbE}$ (Gigabit Ethernet) são comuns em sistemas conectados a Internet[Emmerich et al. 2015]. Toda infraestrutura de rede deve ser avaliada visando uma adequada prestação de serviço, utilizando técnicas de análise. 
A tarefa de monitorar um tráfego elevado com ferramentas de sniffing é exigente, sendo necessário aplicar poder computacional significativo para tal tarefa [Moreno et al. 2015]. Assim são adotados frameworks de captura de pacotes à altas velocidades como solução.

Para pequenos e médios provedores, saber quais Placas de Rede e Framework atendem de forma eficiente as suas necessidades é importante. Já que as companhias provedoras não possuem muitos recursos para serem investidos em poder computacional. Nenhum trabalho pesquisado até o momento realizou uma avaliação de quais fatores possuem mais impacto no tráfego de pacotes de uma rede.

Os poucos trabalhos que realizam avaliações isoladas de cada tecnologia, não consideraram o efeito de múltiplos fatores conjuntamente. Logo, o objetivo deste trabalho é auxiliar gerentes de infraestruturas computacionais na escolha de placas de rede e framework de processamento de tráfego em situações específicas, oferecendo um estudo de desempenho detalhado sobre tais combinações.

Este trabalho está organizado da seguinte forma. A Seção 2 apresenta os trabalhos relacionados. A seção 3 tem-se a análise de sensibilidade realizada e seus resultados obtidos. Por último, as conclusões e trabalhos futuros são apresentados na seção 4.

\section{Trabalhos Relacionados}

Esta seção apresenta alguns trabalhos produzidos acerca de análise de desempenho de NIC's 10GbE ou frameworks de captura de pacote. No Trabalho de [Gallenmüller 2014] um modelo é desenvolvido, demonstrando a relação fundamental entre custos e desempenho destes frameworks de processamento de pacotes. [Gallenmüller 2014] não leva em conta diferentes placas para realizar a análise, se limitando apenas aos frameworks.

Em [Barbette et al. 2015] foi analisado e comparado quantitativamente vários frameworks de E/S de pacotes, mostrando sua superioridade ao encaminhamento baseado em kernel. No trabalho de [Barker 2015] é realizada uma análise de desempenho entre diferentes frameworks. O trabalho não realiza uma análise de quais fatores impactam mais as variáveis respostas, utilizando apenas os Framework de captura de pacotes como fator.

O trabalho de [Gallenmüller et al. 2015] são analisados vários frameworks, na qual é introduzido um modelo para estimar e avaliar o desempenho dessas estruturas de processamento de pacotes. Os autores não realizam medições com diferentes placas de rede como fatores e não realizam uma análise de sensibilidade. O trabalho de [Marcuzzo and dos Santos 2017] foi realizada uma avaliação de desempenho comparativa, utilizando diferentes frameworks como fatores. Os autores porém, não realizaram um DoE juntamente com a análise de sensibilidade dos fatores e o fator placa de rede não foi utilizado.

Este trabalho foi o único trabalho que realizou a análise de sensibilidade entre os demais trabalhos, onde apresentou mais métricas analisadas em relação aos demais trabalhos. Por fim os demais critérios foram se os demais trabalhos utilizaram NIC's e Frameworks como fatores. Este trabalho foi o único que utilizou diferentes combinações de NIC e Framework. 


\section{Análise de Sensibilidade}

Esta seção descreve os experimentos realizados para analisar os fatores que tiveram mais influência na acurácia das métricas analisadas através da realização de uma análise de sensibilidade utilizando as métricas: Throughput, Uso de CPU e Perda de Pacotes.

\subsection{Ambiente de Testes}

Foram adotados dois computadores, com configurações iguais e um aparelho Mikrotik CCR1036-8G-2S+ ${ }^{1}$. Nos experimentos, o Mikrotik - também conhecido como RouterBoard - foi responsável pela geração do tráfego. A medida de envio do tráfego é PPS (pacotes por segundo), na qual o dispositivo gerava uma rajada de pacotes a cada segundo, sendo utilizado o tempo de 60 segundos para a coleta dos resultados. O uso do CCR1036$8 G-2 S+$ é justificado pelos vários parâmetros de rede disponíveis em sua ferramenta de geração de tráfego, denominada Traffic-generator.

Em cada um dos computadores foi instalada uma placa de rede diferente. As placas de rede avaliadas foram especificamente a Intel X520-DA2 e a Chelsio T520-SO-CR. O uso de tais placas é justificada por serem de uso comum pelos provedores da região. Como mencionado anteriormente, os frameworks de leitura e captura de pacotes PF_Ring e Netmap foram adotados, dado a grande quantidade de trabalhos que os utilizam. Para poder realizar a medição das métricas foram definidos três fatores: Placa de Rede, Framework de Captura de Pacotes e Tamanho de Pacote. As variações de tamanho do pacotes são definidas na RFC 2544 que trata da padronização de testes em dispositivos de rede, bem como a utilização do protocolo UDP nos pacotes gerados [Bradner and McQuaid 1999].

\subsection{Throughput}

A Figura 1a apresenta o gráfico de Pareto para os fatores relacionados ao Throughput. O Tamanho do Pacote possui maior impacto dentre todos os fatores, portanto o Tamanho dos Pacotes de um tráfego de dados é determinante na confiabilidade da medição. A Figura $1 \mathrm{~b}$ mostra o gráfico de Efeitos Principais para o fator Throughput. Para atender a um tráfego com as mesmas especificações, a placa da Chelsio consegue atingir uma Taxa de Transferência maior que a placa da Intel. Quanto maior o pacote, mais dados são transportados e portanto maior a vazão ou Throughput necessário para transferi-los. Nota-se que os níveis de pacote 512 bytes e 768 bytes apresentam um grau de interação maior, logo as placas são sensíveis à variação desses níveis, de acordo com a Figura 1c. Ao analisar a relação entre o Throughput médio e o fator Framework observa-se que há interação de todos os níveis do fator Tamanho de Pacote. Com a interação ocorrendo o Throughput médio dos frameworks é dependente das variações dos níveis do fator Tamanho de Pacote.

\subsection{CPU}

Observando a Figura 2a, nota-se que o Tamanho do Pacote possui maior impacto dentre todos os fatores, logo o mesmo é determinante no impacto de consumo de dados da CPU. Os fatores Placa de Rede e Framework não apresentaram significância, no que diz respeito ao impacto ocasionado na acurácia da métrica de Uso da CPU. Os pacotes de tamanho menor apresentaram um consumo de CPU elevado, os pacotes de 64 bytes apresentaram uma média maior, de acordo com o gráfico da Figura $2 \mathrm{~b}$. Os pacotes de tamanho maior

\footnotetext{
${ }^{1}$ https://mikrotik.com/product/CCR1036-8G-2Splus
} 


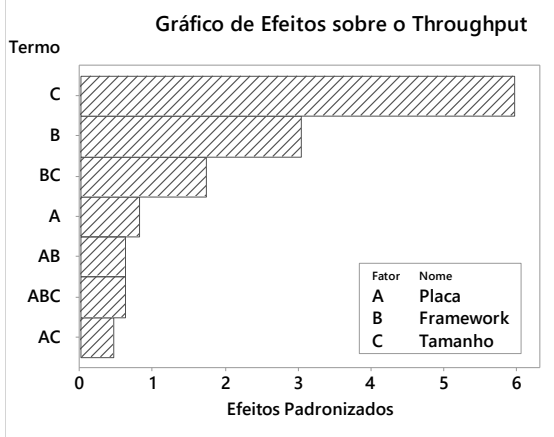

(a) Influência dos Fatores na Acurácia.

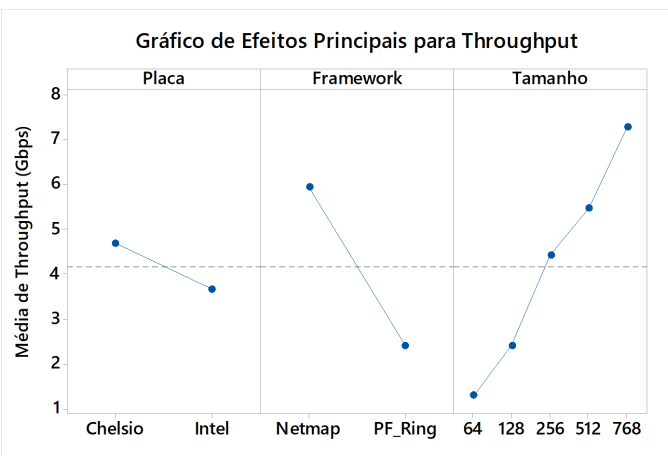

(b) Taxa Média de Throughput em cada Nível dos Fatores.

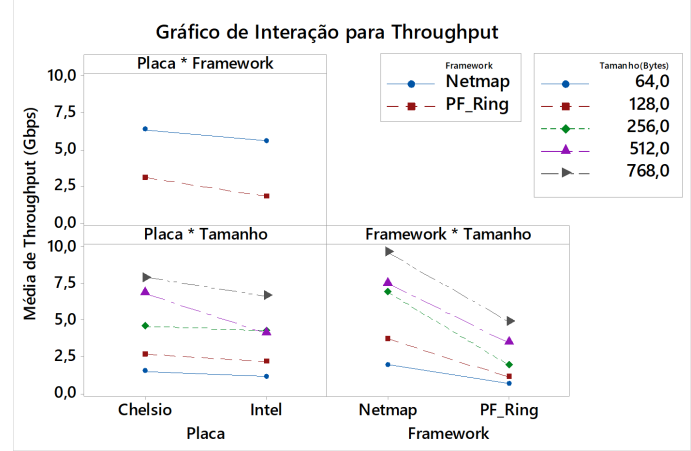

(c) Interação dos Níveis dos Fatores.

Figura 1. Resultado das Medições sobre o Throughput.

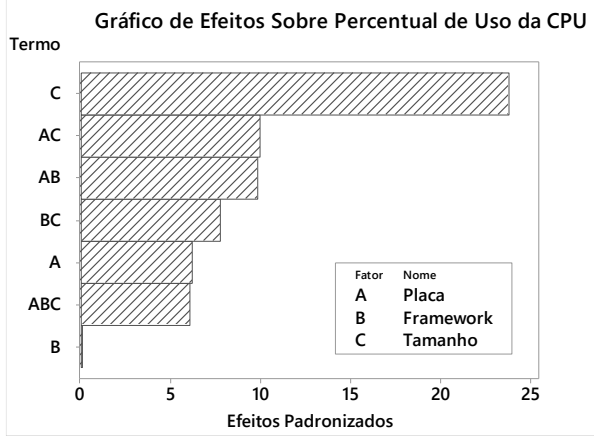

(a) Influência dos Fatores na Acurácia (Uso de CPU).

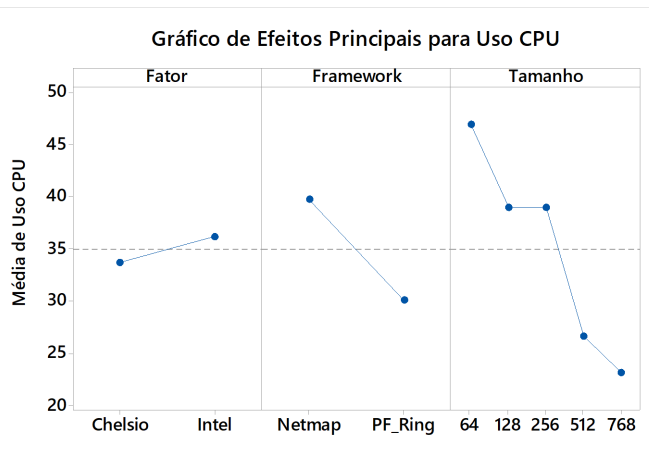

(b) Percentual Médio de Uso de CPU em cada Nível dos Fatores.

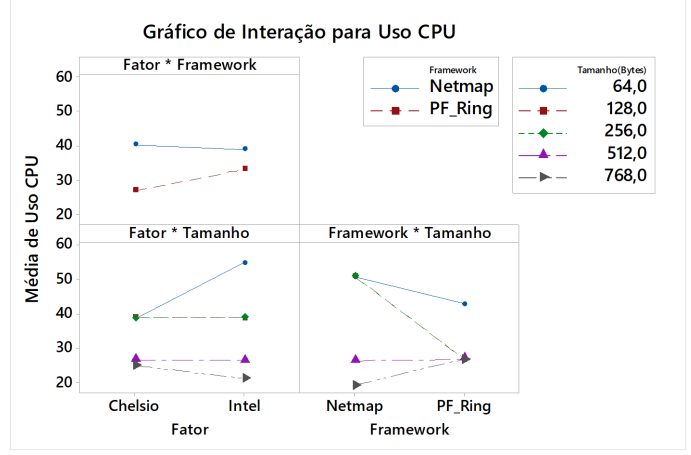

(c) Interação dos Níveis dos Fatores.

Figura 2. Resultado das Medições sobre o Consumo Médio da CPU. 
apresentaram baixa exigência de poder computacional. Cada pacote ao ser recebido pela Placa de Rede e lido pelo Framework tem o seu cabeçalho processado. Quanto mais pacotes chegam a interface de Rede mais CPU é exigida para processar o cabeçalho de cada pacote. A Combinação da Placa da Chelsio com o Framework Netmap, foi a que mais exigiu recursos da CPU, conforme a A Figura 2c. A placa da Chelsio apresenta um consumo de CPU mais eficiente com pacotes menores e intermediários, já para pacotes grandes a escolha pela placa da Intel é a mais acertada. O Framework PF_Ring consome menos CPU com pacotes menores, já para pacotes maiores a tendência é o Netmap ser melhor.

\subsection{Pacotes Descartados}

A Figura 3a apresenta o gráfico de Pareto para os fatores relacionados a variável resposta Pacotes Descartados. O fator Placa de Rede foi o que menos significativo, indicando que esse fator não acarreta em mudanças no experimento. A Figura $3 b$ mostra o gráfico de

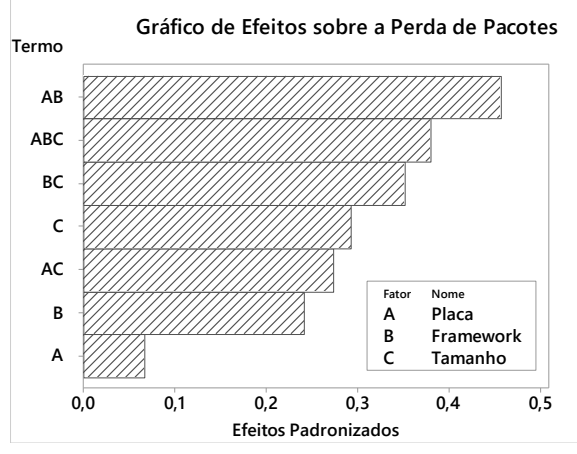

(a) Influência dos Fatores na Acurácia (Perda de Pacote).

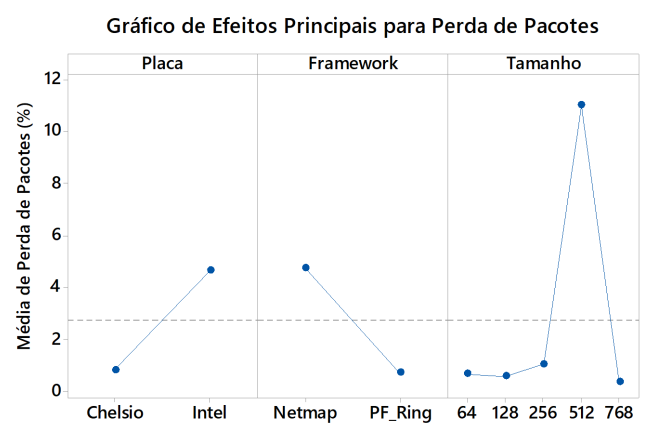

(b) Percentual Médio de Pacotes Descartados em cada Nível dos Fatores.

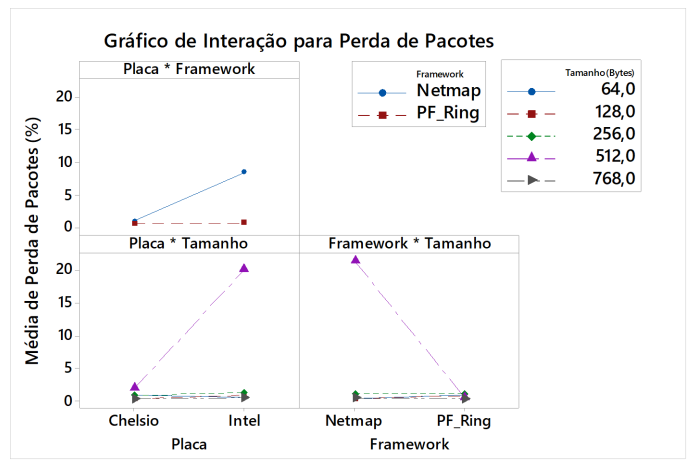

(c) Interação dos Níveis dos Fatores.

Figura 3. Resultado das Medições sobre o Percentual de Pacotes Descartados.

efeitos principais da métrica Pacotes Descartados. Observando o Gráfico nota-se que a variável resposta é afetada pelos níveis dos fatores: Placa de Rede e Framework. A suspeita de que o tamanho de pacote 512 bytes tenha atingido o pico ilustrado na Figura 3 b é algum conflito de configurações entre o Netmap e a Placa Intel. Esse conflito teria causado alguma interferência na alocação dos anéis de memória do Netmap, ocasionando essa perda de pacotes. Atentando-se a Figura 3c, o nível 512 bytes apresentou uma elevada perda de pacotes diante da combinação da placa Intel e o framework Netmap. O 
primeiro gráfico indica que a relação entre a Placa de Rede e o Throughput apresenta pouca dependência do framework PF_Ring já que o mesmo não apresentou interação.

\section{Conclusão}

Este trabalho desenvolveu análises de artefatos de rede (Placas de Rede e Framework de captura e processamento de pacotes). A análise visa auxiliar os administradores de redes, gerentes de infraestruturas, dentre outros profissionais da área a escolher tais artefatos. Em específico as placas de redes Chelsio T520-SO-CR e Intel X520-DA2 foram submetidas a experimentos juntamente com os frameworks Netmap e PF_Ring. Cada situação de uso, levou em conta os impactos dos fatores nas métricas, bem como as interações desses fatores na variável resposta. Portanto, diferentes combinações são indicadas para determinados contextos. Este trabalho teve algumas limitações, uma delas está na quantidade de placas de rede. A utilização de um número maior de Placas de Rede traria mais consistência na validação deste trabalho. No entanto, isso não foi possível devido ao elevado custo financeiro de tais aquisições. Para trabalhos futuros, será utilizado parâmetros que compreendam além da pilha TCP/IP, o próprio driver do adaptador.

\section{Referências}

Barbette, T., Soldani, C., and Mathy, L. (2015). Fast userspace packet processing. In Architectures for Networking and Communications Systems (ANCS), 2015 ACM/IEEE Symposium on, pages 5-16. IEEE.

Barker, S. A. (2015). Comparison of ring-buffer-based packet capture solutions. Technical report, Sandia National Laboratories (SNL-CA), Livermore, CA (United States).

Bradner, S. and McQuaid, J. (1999). Rfc 2544. Benchmarking methodology for network interconnect devices.

Cetic.br (2016). Os provedores de acesso à internet no brasil: Como atuam e qual sua importância para o desenvolvimento da internet brasileira. volume 8. CGI.BR/NIC.BR.

Emmerich, P., Raumer, D., Beifuß, A., Erlacher, L., Wohlfart, F., Runge, T. M., Gallenmüller, S., and Carle, G. (2015). Optimizing latency and cpu load in packet processing systems. In Performance Evaluation of Computer and Telecommunication Systems (SPECTS), 2015 International Symposium on, pages 1-8. IEEE.

Gallenmüller, S. (2014). Comparison of memory mapping techniques for high-speed packet processing. Technical University of Munich.

Gallenmüller, S., Emmerich, P., Wohlfart, F., Raumer, D., and Carle, G. (2015). Comparison of frameworks for high-performance packet io. In Proceedings of the Eleventh ACM/IEEE Symposium on Architectures for networking and communications systems, pages 29-38. IEEE Computer Society.

Marcuzzo, L. d. C. and dos Santos, C. R. (2017). Análise e comparaçao de técnicas e aceleradores de processamento de pacotes. ERRC 2017, page 2.

Moreno, V., Ramos, J., García-Dorado, J. L., Gonzalez, I., Gomez-Arribas, F. J., and Aracil, J. (2015). Testing the capacity of off-the-shelf systems to store $10 \mathrm{gbe}$ traffic. IEEE Communications Magazine, 53(9):118-125. 